\title{
Perspectives on Concealed Questions
}

\author{
Floris Roelofsen and Maria Aloni \\ University of Amsterdam
}

\begin{abstract}
This paper presents a novel theory of concealed questions (CQs). The theory is the first to provide a unified and principled account of definite, indefinite, and quantified CQs and CCQs (CQ-containing CQs).
\end{abstract}

\section{Introduction}

Concealed questions (CQs) are determiner phrases (DPs) which are naturally paraphrased as identity questions.

(1) John knows the capital of Italy.

$\approx$ John knows what the capital of Italy is.

(2) They revealed the winner of the contest.

$\approx$ They revealed who the winner of the contest was.

Concealed questions do not only arise with definite determiner phrases, but also with indefinite and quantified ones.

(3) John knows a doctor who could help you.

(Frana 2006)

(4) John knows every phone number.

(Heim 1979)

Heim (1979) observed that quantified CQs exhibit an interesting ambiguity. If no particular context is given, the most salient reading of (4) is probably what we call the pair-list reading:

(5) John knows that Ann's number is 503, that Bill's number is 431, etc.

But if John's task is to assign to every newly installed phone a number that is not yet taken by any other phone, then he doesn't need to "know every phone number" in the sense of knowing which phone number is whose, but merely in the sense of knowing which numbers are somebody's at all (Heim 1979: p.60). We call this the set-reading:

(6) John knows of every phone number that it is a phone number.

Apart from the basic definite, indefinite and quantified CQs examplified in (1)-(4), there are also structurally more involved cases like:

(7) John knows the capital that Fred knows.

(Heim 1979) 
Heim (1979) observed that such CQ-containing CQs (CCQs) are ambiguous in an interesting way. Sentence (7) has the following two readings:

(8) John and Fred know the same capital.

[Reading A]

(9) John knows which capital Fred knows.

[Reading B]

Suppose Fred knows the capital of Italy. Then on Reading A, (7) entails that John also knows the capital of Italy. On Reading B, (7) lacks this entailment. It only follows that John knows that Fred knows what the capital of Italy is. John himself may lack this knowledge.

Of course, CCQs may also be indefinite or quantified:

John knows a doctor that Fred knows.

John knows every / most / at least one capital that Fred knows.

In the case of quantified CCQs the A/B ambiguities interact with the pair-list/setreading ambiguities. The pertinent readings will be considered in detail below.

Currently, there is no unified account of definite, indefinite, and quantified CQs and CCQs. We will propose such an account shortly, but before doing so we will briefly consider various theoretical options that have been explored in the recent literature. We will point out what we think is the most promising direction to take, and what exactly are the outstanding problems.

\section{Recent Approaches}

Recent accounts of concealed questions differ along two dimensions: ${ }^{1}$

\begin{tabular}{l|cc}
$\begin{array}{l}\text { Questions / } \\
\text { Propositions }\end{array}$ & $\begin{array}{l}\text { Romero, 2007 } \\
\text { Nathan, 2006 }\end{array}$ & Aloni, 2007 \\
Properties & Frana, 2006 & Schwager, 2007 \\
$\begin{array}{l}\text { Individual } \\
\text { concepts }\end{array}$ & Romero, 2005 & Schwager, 2007 \\
\cline { 2 - 2 } & {$[-\mathrm{P}]$} & {$[+\mathrm{P}]$}
\end{tabular}

\footnotetext{
${ }^{1}$ An important contribution that is missing from the chart is (Harris 2007). Harris' work certainly belongs in the $[+\mathrm{P}]$ column, but it is not entirely clear in which row it belongs. Harris analyzes CQs as functions from individual concepts to identity questions, or, equivalently, as functions from world-pairs to sets of individual concepts. Aloni's (2007) work can be seen as an attempt to simplify Harris' analysis.
} 
Along the vertical dimension, which we call the TYPE dimension, theories differ in that they assign different semantic types to concealed questions. Some take CQs to denote propositions, others take them to denote properties or individual concepts, etc. There are two kinds of arguments along the TYPE dimension. The first involves coordination. If concealed questions are not taken to denote propositions it is hard to explain why they can be coordinated with full-fledged declarative and interrogative complement clauses, as in (12) and (13).

(12) They revealed the winner of the contest and that the President of the association would hand out the prize in person.

I only knew the capital of Italy and who won the Worldcup in 1986.

The other argument along the TYPE dimension is one of parsimony. All CQembedding verbs also embed full-fledged declarative and/or interrogative clauses. Thus, if CQs are taken to denote propositions, CQ-embedding verbs can be composed with their CQ-complements just as they would be composed with their fullfledged declarative and/or interrogative complements. If CQs are not taken to denote propositions but, say, properties, then it must be assumed that CQ-embedding verbs are associated with at least two distinct lexical items: one which embeds proposition denoting expressions and one which embeds property denoting expressions. Clearly, such stipulations should be avoided whenever possible. Thus, both arguments along the TYPE dimension favour approaches that take CQs to denote propositions.

Along the horizontal dimension, theories differ in that some take the interpretation of CQs to be dependent on the particular perspective that is taken on the individuals in the domain of discourse, while others do not. What this means exactly is best explained by means of an example.

Suppose that in front of you lie two face-down cards. One is the Ace of Hearts, the other is the Ace of Spades, but you don't know which is which. You have to choose one card: if you choose the Ace of Hearts you win 10 euros, if you choose the Ace of Spades you lose 10 euros. Now consider the following sentence:

You know the winning card.

Is this sentence true or false in the given situation? On the one hand, the sentence is true: you know that the Ace of Hearts is the winning card. On the other hand, the sentence is false: as far as you know, the winning card may be the card on the left, but it may just as well be the card on the right. Intuitively, there are two ways in which the cards may be identified in this situation: by their position (the card on the left, the card on the right) or by their suit (the Ace of Hearts, the Ace of Spades). Whether (14) is judged true or false depends on which of these perspectives is adopted. If identification by suit is adopted, the sentence is judged true. But if identification by position is adopted, the sentence is judged false.

Some theories of CQs account for such perspective-related ambiguities, others do not. Clearly, the ones that do are to be preferred.

This leads us to the conclusion that Aloni's (2007) theory, which analyzes 
concealed questions as proposition denoting expressions and accounts for perspectiverelated ambiguities, is the most promising theory among those proposed in the recent literature. Several problems remain however. In the next section, we will discuss Aloni's theory and the problems it faces.

\section{Concealed Questions under Cover}

Aloni's theory of concealed questions builds on the theory of questions developed in (Aloni 2001). This is a modification of Groenendijk and Stokhof's analysis, especially geared at capturing the perspective-sensitive nature of questions and other operators in natural language. We will first review Aloni's approach to questions in general. Then we will turn to her account of concealed questions in particular, and point out some of its shortcomings.

\subsection{Conceptual Covers}

Consider again the card situation discussed in the previous section. In front of you lie two face-down cards. One is the Ace of Spades, the other is the Ace of Hearts. You don't know which is which. There are two different ways of identifying the two cards in this scenario: by their position on the table (the card on the left, the card on the right) and by their suit (the Ace of Spades, the Ace of Hearts). Aloni (2001) proposes to formalize such methods of identification in terms of conceptual covers. A conceptual cover is a set of individual concepts which exclusively and exhaustively covers the domain of individuals: each individual is identified by exactly one concept in each world. More formally:

Definition. [Conceptual covers] Given a set of possible worlds $W$ and a domain of individuals $D$, a conceptual cover $C C$ based on $(W, D)$ is a set of functions $W \rightarrow D$ such that:

$$
\forall w \in W: \forall d \in D: \exists ! c \in C C: c(w)=d
$$

Illustration. To formalize the card situation discussed above we need a model with two worlds, $w_{1}$ and $w_{2}$, and a domain consisting of two individuals, $\varnothing$ and $\mathbf{\Lambda}$. As illustrated in the diagram below, either $\varnothing$ is the card on the left (in $w_{1}$ ) or it is the card on the right (in $w_{2}$ ).

$$
\begin{array}{llll}
w_{1} & \mapsto & \varnothing & \boldsymbol{\phi} \\
w_{2} & \mapsto \boldsymbol{\phi} & \varnothing
\end{array}
$$

There are only two possible conceptual covers definable over such a model, namely the set A which identifies the cards by their position and the set B which identifies the cards by their suit:

$$
A=\{\text { the card on the left, the card on the right }\}
$$




$$
\mathrm{B}=\{\text { the Ace of Spades, the Ace of Hearts }\}
$$

$\mathrm{C}$ below is a set of concepts which does not constitute a conceptual cover:

$$
\mathrm{C}=\{\text { the card on the left, the Ace of Hearts }\}
$$

For $\mathrm{C}$ to be a conceptual cover, every individual should be identified by exactly one concept in every world. But this is not the case. In $w_{1}$ for example, $\varnothing$ is identified by two concepts, while $\boldsymbol{\beta}$ is not identified by any concept at all.

\subsection{Quantification under Cover}

Aloni (2001) considers a language of first order predicate logic enriched with a question operator ?. A special index $n \in N$ is added to the variables in the language. These indices range over conceptual covers. A model for this language is a quadruple $(W, D, I, C)$ where $W$ is a set of possible worlds, $D$ is a set of individuals, $I$ is a world dependent interpretation function and $C$ is a set of conceptual covers based on $(W, D)$. A conceptual perspective $\wp$ is a function from $N$ to $C$. Sentences are interpreted with respect to assignments under a perspective. An assignment under a perspective $g_{\wp}$ is a function mapping variables $x_{n}$ to concepts in $\wp(n)$, rather than individuals in $D$. Quantification under conceptual cover is defined as follows:

Definition. [Quantification under conceptual cover]

$$
\left[\left[\exists x_{n} \phi\right]\right]_{M, w, g_{\wp}}=1 \text { iff } \exists c \in \wp(n):[[\phi]]_{M, w, g_{\wp}}\left[x_{n} / c\right]=1
$$

On this account, variables range over elements of a conceptual cover, rather than over individuals simpliciter. The denotation of a variable in a world, however, will always be an individual, and never a concept.

Definition. [The denotation of variables] $\left[\left[x_{n}\right]_{M, w, g_{\wp}}=\left(g_{\wp}\left(x_{n}\right)\right)(w)\right.$

The denotation $\left[\left[x_{n}\right]_{M, w, g_{\wp}}\right.$ of a variable $x_{n}$ with respect to a model $M$, a world $w$ and an assignment under a perspective $g_{\wp}$ is the individual $\left(g_{\wp}\left(x_{n}\right)\right)(w)$, i.e., the value of concept $g_{\wp}\left(x_{n}\right)$ in world $w$. On this account, then, variables do not refer to concepts, but to individuals. They do refer, however, in a non-rigid way: different individuals can be their value in different worlds.

\subsection{Questions under Cover}

Questions are analyzed in terms of their possible exhaustive answers, as in (Groenendijk and Stokhof 1984). The evaluation of a question, however, involves quantification over the elements of a conceptual cover rather than over individuals: ${ }^{2}$

\footnotetext{
${ }^{2}$ For simplicity, we only define the semantics of single-constituent questions here (like who called?). See (Aloni 2001) for a generalization, which also deals with multi-constituent questions (like who called whom?).
} 
Definition. [Questions under Cover]

$$
\left[\left[? x_{n} \phi\right]\right]_{M, w, g_{\wp}}=\left\{v \mid \forall c \in \wp(n):\left[[\phi]_{M, w, g_{\wp}\left[x_{n} / c\right]}=[[\phi]]_{M, v, g_{\wp}\left[x_{n} / c\right]}\right\}\right.
$$

To express knowledge- $w h$, the language is extended with a knowledge operator $K_{a}$ selecting questions as complements. A sentence like " $a$ knows- $w h \phi$ " is translated as $K_{a}\left(? x_{n} \phi\right)$. A model for the extended language is a quintuple $(W, D, B e l, I, C)$, where $W, D, I$ and $C$ are as above and $B e l$ is a function mapping individual-world pairs $(a, w)$ into subsets of $W$. Intuitively, $\operatorname{Bel}(a, w)$ represents the belief state of $a$ in $w$. The semantics of the knowledge operator $K_{a}$ is defined as follows:

Definition. [Knowledge-wh] $\left[\left[K_{a}\left(? x_{n} \phi\right)\right]_{M, w, g_{\mathfrak{G}}}=1\right.$ iff $\operatorname{Bel}(a, w) \subseteq\left[? x_{n} \phi\right]_{M, w, g_{\mathfrak{Q}}}$

$K_{a} Q$ is true in $w$ iff $a$ 's belief state is contained in the denotation of $Q$ in $w$. Since $Q$ 's denotation in a world corresponds to Q's true exhaustive answer in that world, $K_{a} Q$ is true in $w$ iff $a$ believes the true exhaustive answer to $Q$ in $w$.

Illustration. Consider again the card situation described above. In front of you lie two face-down cards. One is the Ace of Hearts, the other is the Ace of Spades. You don't know which is which. Furthermore, assume that one of the cards is the winning card, but you don't know which. This situation can be modeled as follows (the dot indicates the winning card):

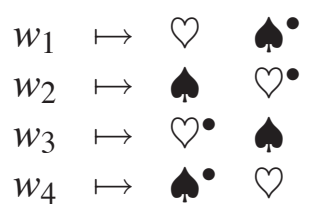

Consider the following interrogative sentence:

a. Which is the winning card?

b. $\quad ? x_{n} \cdot x_{n}=\iota y_{n} P y_{n}$

The evaluation of this sentence depends on the perspective that is taken. There are two possible perspectives. Under one ( $\wp)$, the cards are identified by their position, while under the other $\left(\wp^{\prime}\right)$, the cards are identified by their suit:

$$
\begin{aligned}
& \wp(n)=\{\text { the card on the left, the card on the right }\} \\
& \wp^{\prime}(n)=\text { the Ace of Spades, the Ace of Hearts }
\end{aligned}
$$

The question in (15) partitions the set of worlds in two different ways depending on which perspective is taken:

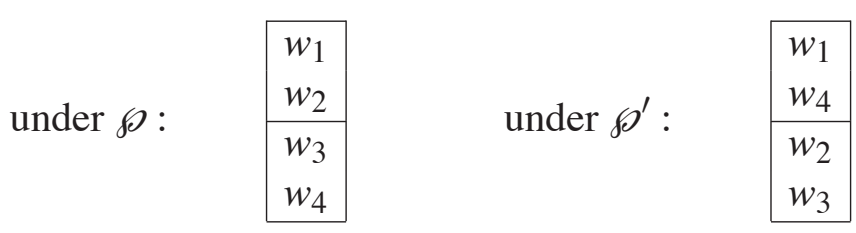


Under $\wp,(15)$ disconnects those worlds in which the winning card occupies a different position. Under $\wp^{\prime}$, it disconnects those worlds in which the winning card is of a different suit. Since different partitions are determined under different perspectives, the fact that different answers are required in different contexts is accounted for. For instance, (16) counts as an answer to (15) only under $\wp^{\prime}$ :

The Ace of Spades is the winning card.

Suppose now you know that the Ace of Spades is the winning card, but you don't know whether it is the card on the left or the one on the right. In this situation your belief state corresponds to the set: $\left\{w_{1}, w_{4}\right\}$. Sentence (17) is then correctly predicted to be true under $\wp^{\prime}$, but false under $\wp$.

a. You know which card is the winning card.

b. $K_{a}\left(? x_{n} \cdot x_{n}=\iota y_{n} P y_{n}\right)$

Aloni (2007) applies this analysis of questions and know-wh to concealed questions.

\subsection{Concealed Questions under Cover}

Aloni assumes that the interpretation of concealed questions crucially involves a type-shifting operator $\uparrow_{n}$ which transforms an entity denoting expression $\alpha$ into the identity question $? x_{n} . x_{n}=\alpha$ (which $x_{n}$ is $\alpha$ ?), where $x_{n}$ ranges over some pragmatically determined conceptual cover.

$$
\uparrow_{n} \alpha={ }_{\mathrm{def}} ? x_{n} \cdot x_{n}=\alpha
$$

Illustrations. Sentence (1), on its CQ-reading, is analyzed as follows:

$$
\begin{array}{ll}
\text { a. John knows the capital of Italy. } & \left(=K_{j}\left(? y_{n} \cdot y_{n}=\iota x_{m} P x_{m}\right)\right) \\
\text { b. } & K_{j}\left(\uparrow_{n} \iota x_{m} P x_{m}\right)
\end{array}
$$

When a question embedding verb like know applies to an entity denoting expression like the capital of Italy, the type-shift rule $\uparrow_{n}$ must apply to avoid type mismatch. The resulting sentence is then interpreted according to the analysis of knowing-wh given in the previous section.

$$
\left[\left[K_{j}\left(? y_{n} \cdot y_{n}=\iota x_{m} P x_{m}\right)\right]\right]_{w, g_{\wp}}=1 \text { iff } \operatorname{Bel}(j, w) \subseteq\left[\left[? y_{n} \cdot y_{n}=\iota x_{m} P x_{m}\right]\right]_{w, g_{\wp}}
$$

The intended reading is obtained if $n$ and $m$ are mapped to the following cover representing identification by name: ${ }^{3}$

$$
n, m \rightarrow\{\text { Berlin, Rome, Paris, } \ldots\}
$$

\footnotetext{
${ }^{3}$ The notation $n, m \rightarrow\{$ Berlin, Rome, Paris, ... $\}$ is used to indicate that the variables $y_{n}$ and $x_{m}$ are taken to range over the cover $\wp(n)=\wp(m)=\{$ Berlin, Rome, Paris, ... $\}$. Notice that the value $\wp(m)$ assigned to $m$ is not really relevant in this case, because $x_{m}$ does not occur free in an intensional context.
} 
Under this resolution the embedded question $? y_{n} \cdot y_{n}=\iota x_{m} P x_{m}$ denotes in $w$ the proposition that Rome is the capital of Italy, if Rome is indeed the capital of Italy in $w$. Sentence (19) then is true in $w$ iff John believes this true proposition in $w$. Example (22) illustrates Aloni's analysis of a quantified CQs:
a. John knows all European capitals.
b. $\forall x_{n}\left(P x_{n} \rightarrow K_{j}\left(\uparrow_{m} x_{n}\right)\right)$

The most natural resolution for $n$ and $m$ here is the following:

$$
\begin{array}{ll}
\text { a. } & n \rightarrow\{\text { the capital of Germany, the capital of Italy, } \ldots\} \\
\text { b. } & m \rightarrow\{\text { Berlin, Rome }, \ldots\}
\end{array}
$$

The sentence is then predicted to be true iff for each European country John knows the true exhaustive answer to the question: What is the capital of that country? This analysis captures the pair-list reading of the sentence. Note that contrary to the previous example, the quantified case crucially requires a shift in perspective, $n$ and $m$ cannot be assigned the same value here, otherwise the quantified questions would be trivialized.

At last we turn to Heim's CCQ example. As we saw in the introduction, Heim (1979) described two readings for sentences like (24), which are usually labeled as Reading A and Reading B.

(24) John knows the capital that Fred knows.
a. John and Fred know the same capital.
[Reading A]
b. John knows which capital Fred knows.
[Reading B]

Aloni analyzes Heim's ambiguity as a de re / de dicto ambiguity:

(25) John knows the capital that Fred knows.
a. $\exists y_{n}\left(y_{n}=\iota x_{n}\left(P x_{n} \wedge K_{f}\left(\uparrow_{m} x_{n}\right)\right) \wedge K_{j}\left(\uparrow_{m} y_{n}\right)\right)$
[Reading A]
b. $\quad K_{j}\left(\uparrow_{n} \iota x_{n}\left(P x_{n} \wedge K_{f}\left(\uparrow_{m} x_{n}\right)\right)\right)$
[Reading B]

The intended readings are captured by assuming the following resolution for the indices $n$ and $m$ :

$$
\begin{array}{ll}
\text { a. } & n \rightarrow\{\text { the capital of Germany, the capital of Italy, ... } \\
\text { b. } & m \rightarrow\{\text { Berlin, Rome }, \ldots\}
\end{array}
$$

On this resolution, Reading A says that there is a unique capital which Fred can identify by name, and that John can identify that capital by name as well. On Reading B, John can only give a descriptive answer to the question what is the capital that Fred knows?. 


\subsection{Problems}

With respect to the data discussed in the introduction, Aloni's theory faces two problems. The first involves quantified CQs such as: John knows every phone number.

As we noted above, quantified CQs are ambiguous between a pair-list reading: John knows that Ann's number is 503, that Bill's number is 431, etc.

and a set-reading:

(29) John knows of every phone number that it is a phone number.

As shown in section 3.4, Aloni's theory accounts for the pair-list reading. But it does not account for the set reading.

The second problem concerns quantified CCQs such as:

$$
\text { John knows every capital that Fred knows. }
$$

These are cases in which the A/B ambiguity that is characteristic for CCQs interacts with the pair-list/set-reading ambiguity that is characteristic for quantified CQs.

Recall that Aloni analyzes the A/B ambiguity of definite CQs such as:

John knows the capital that Fred knows.

as a de re / de dicto ambiguity. On the other hand, her account of quantified CQs assumes a de re representation. In particular, quantified CCQs such as (30) must be represented de re. But this means that the A/B ambiguity of such CCQs cannot be derived as a de re / de dicto ambiguity.

Given certain assumptions, Aloni's theory does provide an alternative strategy to derive the A/B ambiguity, but the required assumptions are not entirely natural and should be avoided if possible. Let us have a closer look.

In a logic assuming quantification under conceptual cover, a de dicto representation always has an equivalent de re representation. This holds also for Aloni's de dicto rendering of $(25) /(31)$. The $\mathrm{A} / \mathrm{B}$ ambiguity must then be captured in terms of different index resolutions. Thus, (31) is represented de re as follows:
a. John knows the capital that Fred knows.
b. $\exists y_{0}\left(y_{0}=\iota x_{1}\left(P x_{1} \wedge K_{f}\left(\uparrow_{2} x_{1}\right)\right) \wedge K_{j}\left(\uparrow_{3} y_{0}\right)\right)$

The A and B readings are obtained by assuming the resolutions in (33) and (34) respectively:

\section{Reading A:}

a. $\quad 0,1 \rightarrow\{$ the capital of Germany, the capital of Italy, ...

b. $2,3 \rightarrow\{$ Berlin, Rome, ... $\}$ 
Reading B:

a. 0 must include the capital that Fred knows;

b. $\quad 1,3 \rightarrow\{$ the capital of Germany, the capital of Italy, ...

c. $2 \rightarrow\{$ Berlin, Rome, ... $\}$

The same strategy could be applied to quantified CCQs like (30):
a. John knows every capital that Fred knows.
b. $\forall x_{1} \forall y_{0}\left(\left(y_{0}=x_{1} \wedge P x_{1} \wedge K_{f}\left(\uparrow_{2} x_{1}\right)\right) \rightarrow K_{j}\left(\uparrow_{3} y_{0}\right)\right)$

The A and B readings can be obtained by assuming the following resolutions:

Reading A:

a. $\quad 0,1 \rightarrow\{$ the capital of Germany, the capital of Italy, ... $\}$

b. $\quad 2,3 \rightarrow\{$ Berlin, Rome, ... $\}$

Reading B:

a. $\quad 0 \rightarrow\{$ the 1 st capital that $\mathrm{F}$ knows, the 2 nd capital that $\mathrm{F}$ knows, ... $\}$

b. $\quad 1,3 \rightarrow\{$ the capital of Germany, the capital of Italy, ... $\}$

c. $\quad 2 \rightarrow\{$ Berlin, Rome, $\ldots\}$

However, the resolution required for reading $\mathrm{B}$ in this case is very implausible. Consider the conceptual cover that must be assigned to 0 in (37a). It is hard to see how such a cover can be constructed or be salient in the described context.

\section{A Pragmatic Theory of Concealed Questions}

We take as our point of departure the pair-list/set-reading ambiguity of quantified CQs. As pointed out above, Aloni's theory fails to account for this ambiguity. In fact, all theories of CQs proposed so far fail to do so. What is it that makes this case so challenging? Consider again the canonical example:

(38) John knows every phone number.

Let us assume (39) as a representation of the truth-conditional meaning of (38), without yet fixing the exact definition of the type-shift operator $\uparrow$.

$$
\forall x_{n}\left(\operatorname{PHONENUmbeR}\left(x_{n}\right) \rightarrow K_{j}\left(\uparrow x_{n}\right)\right)
$$

Now let us just see how $\uparrow$ should be defined to get the facts right. In order to capture the pair-list reading, we need:

$$
\uparrow x_{n} \leadsto ? x_{m} \cdot x_{m}=x_{n}
$$

with:

- $n \rightarrow\{$ Ann's phone number, Bill's phone number, ... 
- $m \rightarrow\{5403,5431, \ldots\}$

In order to get the set reading, we want:

$$
\uparrow x_{n} \leadsto \text { ?PHONENUMBER }\left(x_{n}\right)
$$

with:

- $n \rightarrow\{5403,5431, \ldots\}$

To capture this flexibility, we will assume that $\uparrow$ transforms an entity-denoting expression $\alpha$ into the question $? x_{n} P(\alpha)$, where $P$ is contextually resolved.

$$
\uparrow_{(n, P)} \alpha={ }_{\mathrm{def}} \quad ? x_{n} \cdot P(\alpha)
$$

The type-shift has two pragmatic parameters: $x_{n}$ ranges over some contextually determined conceptual cover and $P$ is a contextually determined property. In ordinary cases $P$ is resolved to the property of being identical to $x_{n}$, i.e., to the "identity property" $\lambda y \cdot y=x_{n}$; or to the property expressed by the CQ noun phrase. In the latter case, the resolution of $n$ becomes irrelevant. Indeed, if $x_{n}$ does not occur free in $\alpha$, then $? x_{n} P(\alpha)$ is equivalent to ?P( $\left.\alpha\right)$, and we will avoid reference to the indexed variable in these cases.

Thus, (38) is now analyzed as:

$$
\forall x_{n}\left(\operatorname{PhONENUmber}\left(x_{n}\right) \rightarrow K_{j}\left(? x_{m} . P\left(x_{n}\right)\right)\right)
$$

The pair-list reading is obtained by resolving $n, m$, and $P$ as follows:

- $n \rightarrow\{$ Ann's phone number, Bill's phone number, ... $\}$

- $m \rightarrow\{5403,5431, \ldots\}$

- $P \rightarrow \lambda y \cdot y=x_{m}$

which yields:

$$
\forall x_{n}\left(\operatorname{Phonenumber}\left(x_{n}\right) \rightarrow K_{j}\left(? x_{m} . x_{m}=x_{n}\right)\right)
$$

The set-reading results from the following resolution:

- $n, m \rightarrow\{5403,5431, \ldots\}$

- $P \rightarrow$ PHONENUMBER

which yields:

$$
\forall x_{n}\left(\operatorname{PHONENUMbeR}\left(x_{n}\right) \rightarrow K_{j}\left(\text { ?PHONENUMBER }\left(x_{n}\right)\right)\right)
$$

In the first case $P$ is resolved to the "identity property", in the second case to the property expressed by the CQ noun phrase. The resolution of $m$ is only relevant in the first case. Notice that, at least for this example, the proposed account does not overgenerate. The only salient covers are Ann's phone number, Bill's phone 
number, ... $\}$ and $\{5403,5431, \ldots\}$. The only salient properties are the identity property, naturally assumed to be always salient, and PHONENUMBER. As a result, any resolution of $n, m$ and $P$ will yield either the pair-list or the set-reading.

Thus, the proposed type-shift approach is flexible enough to account for the pair-list/set ambiguity, and it is the first account of concealed questions to do so.

\subsection{Resolution and Cover Selection}

The proposed account crucially depends on how conceptual covers are selected and on how the property $P$ is resolved. This section discusses some factors that play an important role in these processes.

Cover Selection. As discussed in detail by Aloni (2001), the selection of conceptual covers is subject to general pragmatic constraints. In particular, the selected cover must be salient and yield an interpretation that is relevant, informative, and consistent with respect to the given common ground.

Moreover, there are general preferences for certain covers over others. In particular, the naming cover and the rigid cover-if available-are generally preferred over any other cover. Apart from these covers, which we call basic covers, the interpretation of CQs also often involves what we call derived covers. Given a basic cover $C$ and a relation $R_{\langle s\langle e\langle e, t\rangle\rangle\rangle}$, the derived cover based on $C$ and $R$ is defined as follows:

$$
\{x \mid \exists c \in C . \forall w \cdot R(w)(x(w))(c(w))\}
$$

For example, $\{$ the capital of Italy, the capital of Germany,... $\}$ is a derived cover based on $\{$ Italy, Germany,... $\}$ and the capital-of relation.

Notice that, strictly speaking, derived covers do not necessarily fit the definition of conceptual covers. To see this consider the derived cover the price of milk, the price of juice,... $\}$ which is based on $\{$ milk, juice,... $\}$ and the price-of relation. The crucial observation is that the price of milk and the price of juice may very well pick out the same individual in some possible worlds - a violation of the uniqueness requirement of conceptual covers.

Relational nouns like price and capital make derived covers particularly salient. This explains the observation often made in the literature that CQ head nouns are often relational nouns (see especially Nathan 2006).

Resolution of $P$. We mentioned above that $P$ is typically resolved either to the identity property or to the property expressed by the CQ noun phrase. There are at least two cases, however, in which $P$ may be resolved differently. The first is when the CQ noun phrase is a complex noun phrase. To see this, consider the following situation. In a company every employee has two phone numbers, an old one for external and internal use and a new one only for internal use. John was the phone administrator before the introduction of the new numbers. Now consider the following sentence:

$$
\text { John knows every old phone number. }
$$


In this situation, example (47) has what we call a weak set-reading. On this reading the sentences does not entail that John knows of each old phone number that it is an old phone number, but only that it is a phone number. Recall that (47) is analyzed as follows:

$$
\left.\forall x_{n}\left(\operatorname{OLD}-\operatorname{PHONENUMBER}\left(x_{n}\right) \rightarrow K_{j}\left(? x_{m} \cdot P\left(x_{n}\right)\right)\right)\right)
$$

The weak set-reading results from the following resolution:

- $n, m \rightarrow\{5403,5431, \ldots\}$

- $P \rightarrow$ PHONE-NUMBER

Notice in particular that $P$ is resolved to PHONE-NUMBER and not to OLD PHONENUMBER. The general upshot of this example is that $P$ may be resolved to the CQ head noun rather than the entire CQ noun phrase. We have not found examples in which $P$ can be resolved to parts of a complex CQ noun phrase that do not include the head noun. In (47) for example, $P$ cannot be resolved to OLD. ${ }^{4}$

A second case in which $P$ is not resolved to the identity property or to the CQ noun phrase is when another property is made particularly salient in the given context. Consider the following situation. Tomas is confronted with the following list of South American cities:

$$
\text { Caracas, Montevideo, Lima, Porto Alegre, Quito, Arequipa. }
$$

He is challenged to say which of these cities are capitals and which are not. His wife Tereza can then report:

He only knew the city we visited on our honeymoon last year.

To get this reading, $P$ must be resolved to CAPITAL and not to CITY WE VISITED ON OUR HONEYMOON LAST YEAR. This example shows that $P$ must really be determined pragmatically and cannot be derived compositionally. ${ }^{5}$

This said, however, the generalization that $P$ is typically resolved to the identity property or to the CQ noun phrase remains, of course, intact.

Contrast. Cover selection and the resolution of $P$ are both subject to a contrastrelated constraint. To see this, consider the following example:

John knows every capital, but Bill does not know every capital.

This sentence contrasts/compares John and Bill's respective knowledge. The crucial observation is that in such constructions, the CQ in the first clause and the CQ in the second clause must either both receive a pair-list reading or both receive a setreading. Mixed readings are impossible.

\footnotetext{
${ }^{4}$ This might be related to Gupta's (1980) account of nouns as sorts. On this account, nouns differ from verbal and adjectival predicates in that they contribute principles of transworld identity.

${ }^{5}$ The example also shows that $P \alpha$ should be within the scope of the question operator (contra Heim (1979) and Frana (2006)): Tereza seems to report that Tomas knew whether the city they visited on their honeymoon was a capital, not necessarilly that it was a capital.
} 
The constraining effect of contrast on resolution is familiar from the literature on ellipsis, and may be formalized in terms of focus values (cf. Rooth 1992).

It also plays a crucial role in the interpretation of CCQs. Consider the following example:

John knows every capital that Fred knows.

This quantified CCQ is analyzed as:

$$
\forall x_{m}\left(\left(\operatorname{CAPITAL}\left(x_{m}\right) \wedge K_{f}\left(? x_{h} \cdot P_{1}\left(x_{m}\right)\right)\right) \rightarrow K_{j}\left(? x_{n} \cdot P_{2}\left(x_{m}\right)\right)\right)
$$

Now if (52) is taken to say something about what John knows in contrast with what Fred knows, then $P_{1}$ and $P_{2}$ must be resolved to the same property and $x_{n}$ and $x_{h}$ must be taken to range over the same conceptual cover.

The exact readings of (52) will be discussed in section 4.2 right below. For now, the point of this example is merely to illustrate that contrast may play a significant role in the interpretation of concealed questions.

\subsection{Quantified CCQs}

Let us now consider the case of quantified CCQs in more detail. The interaction between A/B ambiguities and pair-list/set-reading ambiguities results in many different readings. As pointed out above, some of these readings are problematic for Aloni's (2007) account. In fact, other theories in the literature do not fare better in this respect. The pragmatic theory proposed here seems to finally give us a handle on this issue. Consider the standard example again:

$$
\text { John knows every capital that Fred knows. }
$$

and its analysis:

$$
\forall x_{m}\left(\left(\operatorname{CApitaL}\left(x_{m}\right) \wedge K_{f}\left(? x_{h} \cdot P_{1}\left(x_{m}\right)\right)\right) \rightarrow K_{j}\left(? x_{n} . P_{2}\left(x_{m}\right)\right)\right)
$$

Let us first observe on a general level that the sentence may either be taken to say something about John's knowledge in contrast/comparison with Fred's knowledge or it may be taken to say something about John's knowledge about Fred's knowledge. Notice that this distinction corresponds to the Reading A/ Reading B distinction. Let us first consider the case in which (54) is taken to say something about John's knowledge in contrast with Fred's knowledge.

On a more fine-grained level, then, there are again two interpretive options. On the one hand the sentence can be taken to mean that for every country which is such that Fred knows its capital, John also knows its capital (a pair-list reading). On the other hand, the sentence can also be taken to mean that for every capital of which Fred knows that it is a capital, John also knows that it is a capital (a set-reading).

These readings correspond to the following resolutions: 
$\underline{\text { Reading A-pair-list: }}$

- $m \rightarrow\{$ the capital of Italy, the capital of Germany, ...

- $h, n \rightarrow\{$ Rome, Berlin, Paris, $\ldots\}$

- $P_{1} \rightarrow \lambda y \cdot y=x_{h} \& P_{2} \rightarrow \lambda y \cdot y=x_{n}$

Reading A-set:

- $m, n, h \rightarrow\{$ Rome, Berlin, Paris, $\ldots\}$

- $P_{1}, P_{2} \rightarrow$ CAPITAL

Notice that in both cases $P_{1}$ and $P_{2}$ are resolved to the same property and $x_{h}$ and $x_{n}$ range over the same cover, which is as it should be if (54) is interpreted as a contrastive statement.

Now let us consider the Reading B type readings, which say something about John's knowledge about Fred's knowledge. Again, on a more fine-grained level there are two options. First, the sentence can be taken to mean that for every country $x$ such that Fred knows $x$ 's capital, John knows that $x$ is a country such that Fred knows its capital (a pair-list reading). On the other hand the sentence can also be taken to mean that for every country $x$ such that Fred knows that $x$ 's capital is a capital, John knows that $x$ is a country such that Fred knows that $x$ 's capital is a capital (a set-reading).

These readings correspond to the following resolutions:

$\underline{\text { Reading B-pair-list: }}$

- $m, n \rightarrow\{$ the capital of Italy, the capital of France, $\ldots\}$

- $h \rightarrow\{$ Rome, Berlin, Paris, ... $\}$

- $P_{1} \rightarrow \lambda y . y=x_{h}$

- $P_{2} \rightarrow \lambda x_{m} . \operatorname{Capital}\left(x_{m}\right) \wedge K_{f}\left(? x_{h} . P_{1}\left(x_{m}\right)\right)$

Reading B-set:

- $m, n, h \rightarrow\{$ Rome, Berlin, Paris, $\ldots\}$

- $P_{1} \rightarrow$ CAPITAL

- $P_{2} \rightarrow \lambda x_{m} . \operatorname{CAPITAL}\left(x_{m}\right) \wedge K_{f}\left(? x_{h} . P_{1}\left(x_{m}\right)\right)$

Thus, the pragmatic theory proposed here accounts for the four-way ambiguity of quantified CCQs in a straightforward and perspicuous way.

In the next section we turn to an interesting observation made by Greenberg (1977). The present proposal explains the observation and several related facts. 


\section{Greenberg's Observation}

Consider the following minimal pair:

John found out the murderer of Smith.

Sentence (56) contains a concealed question whereas (57) contains an explicit question. Greenberg observed that there is a subtle difference in meaning between the two: (56) necessarily entails that John found out of the murderer of Smith that he murdered Smith (direct reading); (57) does not necessarily have this entailmentit may be used to report, for example, that John found out that his gardener was a much sought-after criminal known as "The Strangler" even if John did not find out that the guy actually murdered Smith (indirect reading). This observation is considered to be an important argument against equating concealed questions with their explicit counterparts, or more generally, against analyzing CQs as questions (cf. Heim 1979, Frana 2006). However, given the right context, many exceptions to Greenberg's observation can be found.

First exception. Let us first point out that the contrast between (56) and (57) is not as strong as Greenberg claimed it to be. In particular, it is not true that (56) necessarily entails that John found out that the murderer of Smith indeed murdered Smith. Consider the following situation. There are ten men in a room, three of them are murderers. John has to find out which of the men are murderers. Now consider the following sentence:

(58) So far, John only found out the murderer of Smith.

In the given context, this sentence does not imply that John found out of the murderer of Smith that he was the murderer of Smith, but merely that he was a murderer.

Second exception. In section 4.1 we pointed out that in sufficiently marked contexts, the property $P$ can be resolved to a property that has nothing to do with the CQ noun phrase. The kind of example that Greenberg uses is no exception to this rule. To see this consider the context described above: ten men in a room, three of them murderers, John must find out which men are murderers. Now take the following sentence:

So far, John only found out the guy with the broken hip.

On its most natural reading, this sentence says that John found out that the guy with the broken hip was one of the murderers. Crucially, it does not entail that John found out that the guy with the broken hip had a broken hip.

Last exception. We have actually already seen another phenomenon which is in conflict with Greenberg's observation: the A reading of CCQs. Consider the standard example of a definite CCQ:

(60) John knows the capital that Fred knows. 
Taking Greenberg's observation seriously, we should expect that the sentence entails that John knows of the capital that Fred knows that it is the capital that Fred knows. But we have already seen that this is not necessarily so. There is a reading of (60), namely Reading A, on which John and Fred know the same capital, but John does not necessarily know that this is the capital that Fred knows.

Note that these counterexamples are hard, if not impossible, to explain on a structural account of Greenberg's contrast (cf. Frana 2006), and suggest a more flexible explanation. In our theory, (56) is analyzed as follows:

$$
\exists x_{m}\left(x_{m}=\iota x_{m}\left(\text { MURDERER-OF-SMith }\left(x_{m}\right)\right) \wedge F_{j}\left(? x_{n} . P\left(x_{m}\right)\right)\right)
$$

In a neutral context, there will be a strong preference for resolving $P$ to the property expressed by the CQ noun phrase, i.e., MURDERER-OF-SMITH. This explains Greenberg's original observation. But in sufficiently marked contexts, $P$ may be resolved differently. This explains the exceptions just noted.

\section{Conclusion}

We have proposed a pragmatic theory of concealed questions whose central assumption is that an entity-denoting expression $\alpha$ may be type-shifted into the question $? x_{n} P(\alpha)$, where $P$ is contextually resolved. The result of the pragmatic resolution is either (1) an identity question: which $x_{n}$ is $\alpha$ ? (with $x_{n}$ ranging over some contextually determined conceptual cover) or (2) the question whether some other contextually determined property $P$ holds of $\alpha$. The first component originates from Aloni's (2007) account, the second component is reminiscent of Heim's (1979) pragmatic approach. The theory is also related in an interesting way to the theory of anaphora proposed in (Roelofsen 2008). In particular, Roelofsen argues that the interpretation of NP and VP anaphora, just like that of concealed questions, crucially involves the contextual resolution of a property $P$.

The theory proposed here is the first to provide a unified account of definite, indefinite and quantified CQs and CCQs. It also provides a novel account of Greenberg's (1977) observation, and several related facts.

\section{References}

Aloni, M. (2001). Quantification under Conceptual Covers. Doctoral disssertation, University of Amsterdam.

Aloni, M. (2007). Concealed questions under cover. To appear in 'Grazer Philosophische Studien'vol. 77.

Frana, I. (2006). The de re analysis of concealed questions. In C. Tancredi, M. Kanazawa, I. Imani, and K. Kusumoto, editors, Proc. of SALT XVI.

Greenberg, B. (1977). A semantic account of relative clauses with embedded question interpretations. Manuscript, UCLA. 
Groenendijk, J. and Stokhof, M. (1984). Studies on the Semantics of Questions and the Pragmatics of Answers. Doctoral disssertation, University of Amsterdam.

Gupta, A. (1980). The Logic of Common Nouns. Yale University Press.

Harris, J. (2007). Revealing concealment: a (neuro-)logical investigation of concealed questions. MA thesis, University of Amsterdam.

Heim, I. (1979). Concealed questions. In R. Bäuerle, U. Egli, and A. von Stechow, editors, Semantics from Different Points of View. Springer, Berlin.

Nathan, L. (2006). On the Interpretation of Concealed Questions. Doctoral disssertation, MIT.

Roelofsen, F. (2008). Anaphora Resolved. Doctoral disssertation, University of Amsterdam.

Romero, M. (2005). Concealed questions and specificational subjects. Linguistics and Philosophy, 28(5), 687-737.

Romero, M. (2007). Connectivity in a unified analysis of specificational subjects and concealed questions. In C. Barker and P. Jacobson, editors, Direct Compositionality. Oxford University Press.

Rooth, M. (1992). Ellipsis redundancy and reduction redundancy. In S. Berman and A. Hestvik, editors, Proceedings of the Stuttgart Workshop on Ellipsis.

Schwager, M. (2007). Keeping prices low: an answer to a concealed question. In A. Gronn, editor, Proceedings of Sinn und Bedeutung XII. 